\title{
Seafloor Acoustic Remote Sensing with Multibeam Echo-Sounders and Bathymetric Sidescan Sonar Systems
}

\author{
CHRISTIAN DE MOUSTIER' and HARUYOSHI MATSUMOTO ${ }^{2}$ \\ ${ }^{1}$ Marine Physical Laboratory, Scripps Institution of Oceanography, 9500 Gilman Drive, La Jolla, California 92093-0205, U.S.A. \\ ${ }^{2}$ Cooperative Institute for Marine Resource Studies, Oregon State University, 2030 Marine Science Drive, Newport, Oregon 97365, U.S.A.
}

(Received 22 January, 1991; accepted 31 March 1992)

Key words: Sonar, remote sensing, acoustic backscatter, seafloor, bathymetry

\begin{abstract}
This paper examines the potential for remote classification of seafloor terrains using a combination of quantitative acoustic backscatter measurements and high resolution bathymetry derived from two classes of sonar systems currently used by the marine research community: multibeam echo-sounders and bathymetric sidescans sonar systems. The high-resolution bathymetry is important, not only to determine the topography of the area surveyed, but to provide accurate bottom slope corrections needed to convert the arrival angles of the seafloor echoes received by the sonars into true angles of incidence. An angular dependence of seafloor acoustic backscatter can then be derived for each region surveyed, making it possible to construct maps of acoustic backscattering strength in geographic coordinates over the areas of interest. Such maps, when combined with the highresolution bathymetric maps normally compiled from the data output by the above sonar systems, could be very effective tools to quantify bottom types on a regional basis, and to develop automatic seafloor classification routines.
\end{abstract}

\section{Introduction}

Applications of acoustics to seafloor remote sensing include measurements such as: bathymetry, providing quantitative information on the relief; acoustic imaging, which is most often used in a qualitative sense for geomorphology and for feature detection of obstacle avoidance; and acoustic bottom loss which is related to the physical properties of the substrate.

Today, bathymetric measurements and seafloor acoustic imaging are usually performed with swath mapping sonars using either the multibeam technology or the phase interferometry technique (Matsumoto, 1990; de Moustier, 1988; Davis et al., 1986). The latter derives bathymetry from differential phase measurements of the seafloor echoes received by at least two rows of transducers spaced some distance

Marine Geophysical Researches 15: 27-42, 1993.

(C) 1993 Kluwer Academic Publishers. Printed in the Netherlands. apart, and it uses the amplitude of these echoes to form a sidescanned acoustic image of the bottom. Because of the directivity of the transducers used in such systems, typically $1^{\circ}$ to $2^{\circ}$ 'along-track' and over $50^{\circ}$ 'across-track', the spatial resolution of acoustic signals backscattered by the seafloor is limited by the bandwidth of the sonar system and by potential ambiguities due to signals arriving at the same time from different areas of the seafloor. By contrast, multibeam echo-sounders rely on the high angular resolution of individual narrow beams, typically $1^{\circ}$ to $3^{\circ}$, to measure depth simultaneously at discrete angular intervals athwartships, thereby avoiding most of the time ambiguities encountered in the sidescan geometry. For the same reason, multibeam echo-sounders can be used to obtain geometrically correct acoustic images of the seafloor (de Moustier, 1968a; Hammerstad et al., 1991).

The raw information collected by both classes of sonar systems is seafloor acoustic backscatter versus time and angle of arrival, and the character of these signals is dictated, for the most part, by the material properties of the substrate and by the micro-relief in the area insonified. Therefore, it should be possible to use the information for remote classification of seafloor types. Such classification has important applications for geomorphology, as well as in offshore mineral resources assessment, within a nation's Exclusive Economic Zones or in the open ocean (e.g. for manganese nodule prospecting) (NRC, 1989). It is also important in sonar modeling work aimed at predicting the behavior of sound at seafloor boundaries. 
Compared to the various attempts that have been made to derive quantitative seafloor acoustic backscatter information from conventional sidescan sonar systems (e.g. Mitchell and Somers, 1989), in the following we show that high-resolution swath bathymetry is a key ingredient in the solution. We address acoustic measurements made with multibeam echo-sounders in the first section and devote the second section to the bathymetric sidescan sonar approach.

\section{Acoustic Measurements with Multibeam Echo-sounders}

The majority of existing multibeam echo-sounders use a set of hull-mounted transducers and intersecting fan-shaped beams to measure depth with better than $0.5 \%$ accuracy at angular increments of $1^{\circ}$ to $4^{\circ}$, over angular sectors ranging from $+/-20^{\circ}$ to $+1-70^{\circ}$ on either side of vertical. Operating acoustic frequencies of existing systems range from 12 to $15 \mathrm{kHz}$ for full ocean depth and 30 to $200 \mathrm{kHz}$ for coastal and shallow water environments (de Moustier, 1988). Although the following discussion is based on results obtained with a $12 \mathrm{kHz}$ Sea Beam multibeam echo-sounder, the techniques used and the underlying assumptions are the same for other systems. Higher acoustic frequencies will provide increased spatial resolution over shorter athwartships ranges.

The Sea Beam system transmits a $7 \mathrm{~ms}$ pulse of $12.158 \mathrm{kHz}$ over a beam roughly $2.66^{\circ}$ wide in the fore-aft direction, and $54^{\circ}$ wide athwartships. It receives with a set of 16 preformed beams, nominally $2.66^{\circ}$ wide athwarthsips, and $2.66^{\circ}$ apart. A typical echo sequence for this system is shown in Figure 1. These data were recorded with a data acquisition system designed and built at the Marine Physical Laboratory (de Moustier and Pavlicek, 1987) to record base-banded quadrature samples of the acoustic waveforms for each preformed beam, at the output of the analog beamformer of the Sea Beam system. The echo processor unit of this sonar system determines the time of arrival of echoes received for each beam, and converts this information to depth and horizontal distances across track. These data are in turn displayed as a swath of bottom contours along the ship's track.
To derive quantitative information from these recorded waveforms, several approaches are available: (1) the probability distribution function of the peak amplitude of near-normal incidence returns, taken over a number of successive pings, gives indication of the seafloor micro-roughness in the area insonified (Stanton, 1984; de Moustier, 1986b); (2) statistics of the instantaneous amplitude and phase samples gives clues to the distribution of scattering centers on the bottom (Ol'shevskii, 1967; Alexandrou et al., 1992); (3) the shape of the angular dependence function for seafloor acoustic backscatter is related to the physical processes (e.g. surface roughness, volume scattering in sediments) responsible for the backscattered sound (Jackson et al., 1986); and (4) acoustic backscatter coefficients computed on each beam are indicative of the bottom type. In the following, the steps required to derive these coefficients are briefly described. A detailed version of this methodology is available elsewhere (de Moustier and Alexandrou, 1991).

Starting from the preformed waveforms whose envelopes are shown in Figure 1a, and assuming that DC offset as well as gain differences between channels have been resolved, the first step is to remove the sidelobe interference inherent to the multibeam geometry (Figure $1 \mathrm{~b}$ ). This procedure works on the complex representation of the waveform and applies conventional adaptive noise cancelling techniques to remove the part of the echo for which there is correlation between a reference beam, usually a specular return, and the other beams (Alexandrou and de Moustier, 1988).

The centroid of the echo received by each beam is then determined. This point is assumed to coincide with the maximum response axis of the beam so that the beam pattern function and the area insonified can be used to compute a normalized power for each return. This assumption is reasonable whenever an echo is detectable above the noise. An example of the result is shown in Figure 2, where the echo envelopes for one ping are displayed with their corresponding theoretical insonification function. These functions are determined for each beam by propagating the transmitted pulse, over the area delimited by the intersection of the roll corrected beam and the seafloor whose athwartships slope has been estimated as described below. The beam patterns are computed using the actual sonar geometry, taking 


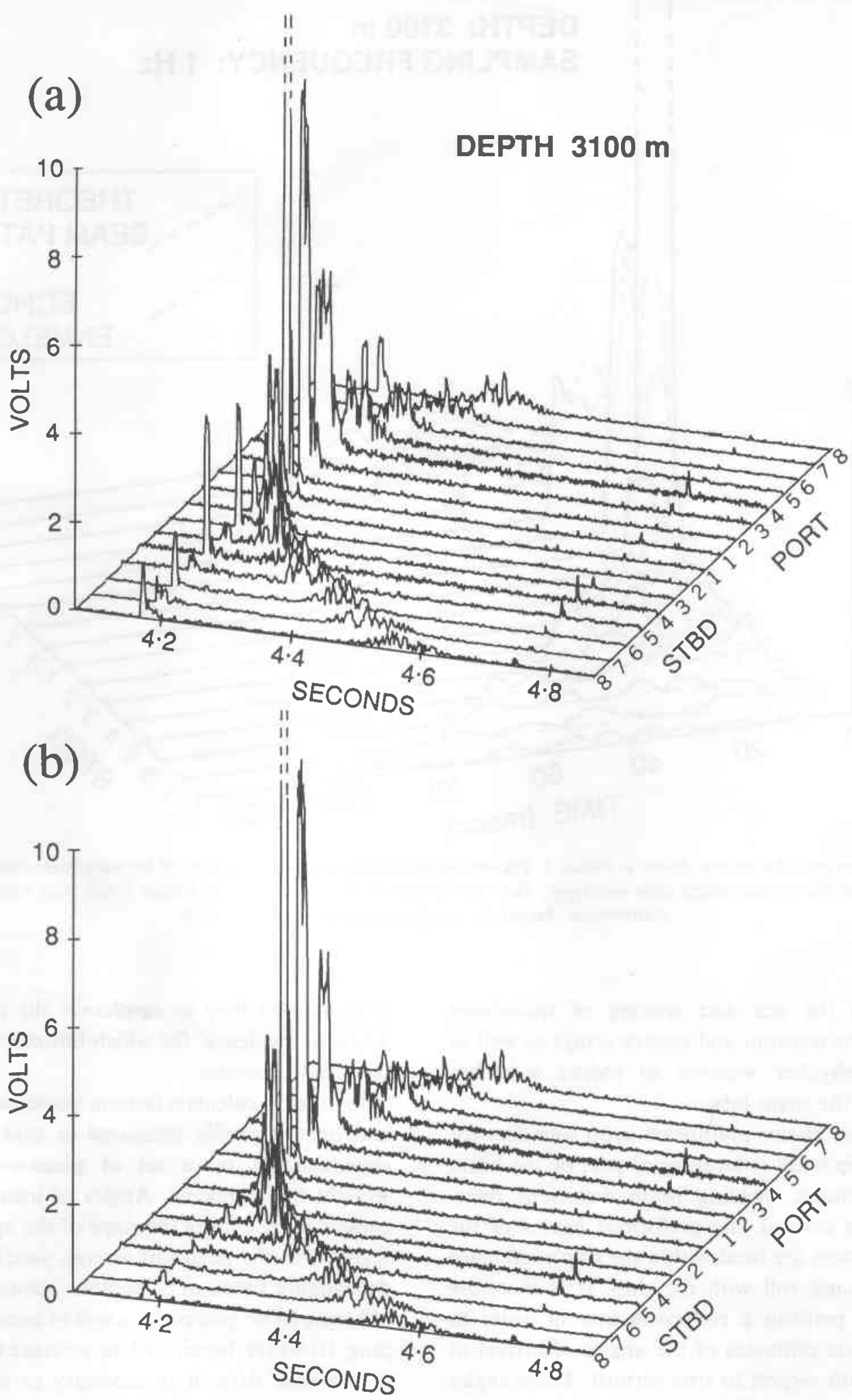

Fig. 1. Envelopes of the echo signals base-banded and quadrature sampled at $1 \mathrm{kHz}$. The time axis represents time elapsed since sonar transmission. Beams are numbered 1 to 8 from the ship's vertical axis out. Amplitude is expressed as a relative voltage. (a) Envelopes of signals as they appear at the output of the beam-former. (b) Envelopes of the signals after sidelobe filtering. 


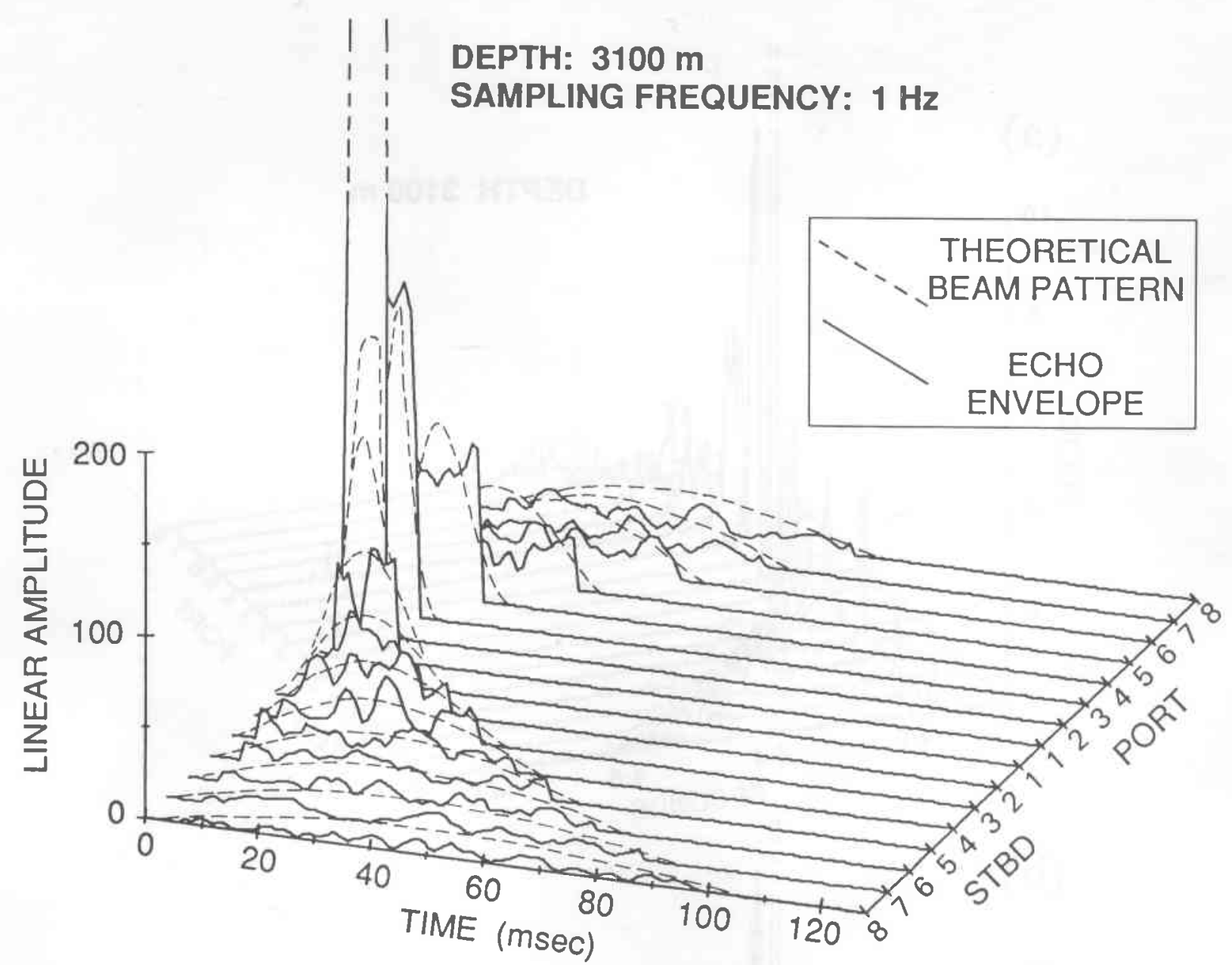

Fig 2. Blow up of the echo returns shown in Figure 1. Theoretical beam pattern functions computed for each beam direction are overlaid (dashed lines) on the corresponding echo envelopes. The time spread of the echoes seen in Figure 1 has been removed for plotting convenience. Amplitude is expressed in relative linear units.

into account the size and spacing of transducer elements in the transmit and receive arrays as well as a Dolph-Chebychev window to reduce sidelobes $30 \mathrm{~dB}$ below the main lobe.

Unlike more recent multibeam echo-sounders for which the ship's roll is an integral part of the beam formation process, yielding beam directions referenced to true vertical, the preformed beams of the Sea Beam system are fixed within the ship's reference frame and hence roll with the ship. It is therefore necessary to perform a roll correction in order to obtain accurate estimates of the angles of arrival of the echoes with respect to true vertical. These angles of arrival must be further corrected to account for raybending effects due to variable sound velocity through the water column. The term 'angle of ar- rival' is used here to emphasize the difference with 'angle of incidence' for which bottom slopes must be taken into account.

In order to calculate bottom slopes across track, the bathymetric profile measured at each ping cycle is approximated by a set of piece-wise continuous straight line segments. Angles of incidence are then computed by adding the slope of the appropriate line segment to the angles of arrival, yielding an angular dependence function of seafloor acoustic backscatter (10 $\log _{10}$ (echo power) vs. angle of incidence) for each ping. However, because of the variance typically found in recorded data, it is necessary to average several pings before a 'representative' angular dependence function can be obtained. This also requires that the data be recorded over a homogeneous seafloor area. 
As an example, a data set consisting of 300 pings recorded with the Sea Beam system over Magellan Rise (near $7^{\circ} 04^{\prime} \mathrm{N}, 176^{\circ} 53^{\prime} \mathrm{W}$ ) is presented here. These data represent one swath delimiting a small area, about $4 \mathrm{~km}^{2}$, that is uniformly covered with foraminiferal ooze, and whose bathymetry varies by less than $20 \mathrm{~m}$ overall at an average depth of $3150 \mathrm{~m}$ as seen in Figure 3. These data were recorded while the ship advanced at about 2 knots $\left(1 \mathrm{~m} \mathrm{~s}^{-1}\right)$ and bathymetry is displayed using a 10 ping average. The overall angular dependence function of seafloor acoustic backscatter at $12 \mathrm{kHz}$ for this area is shown in Figure 4. The top curve represents the 'raw' version in which the mean echo power received in each beam has been binned in $1^{\circ}$ angular bins according to the original roll compen- sated beam direction, and averaged over the number of samples in each bin. Although the function spans from $-20^{\circ}$ to $+20^{\circ}$ incidence, for this plot, it is assumed to be symmetric (flat bottom) and it is folded about the vertical to a one-sided function. The middle curve shows the effect of a cross-track bottom slope correction on each ping, making it clear that the flat bottom assumption is inadequate even for such low relief. The bottom curve shows the effect of the combined bottom slope and area insonified corrections, reinforcing the need to account for the larger footprint of the outer beams compared to the near-nadir beams. All three curves have been normalized to their respective values at normal incidence and they are displayed together for comparison only. Absolute backscattering

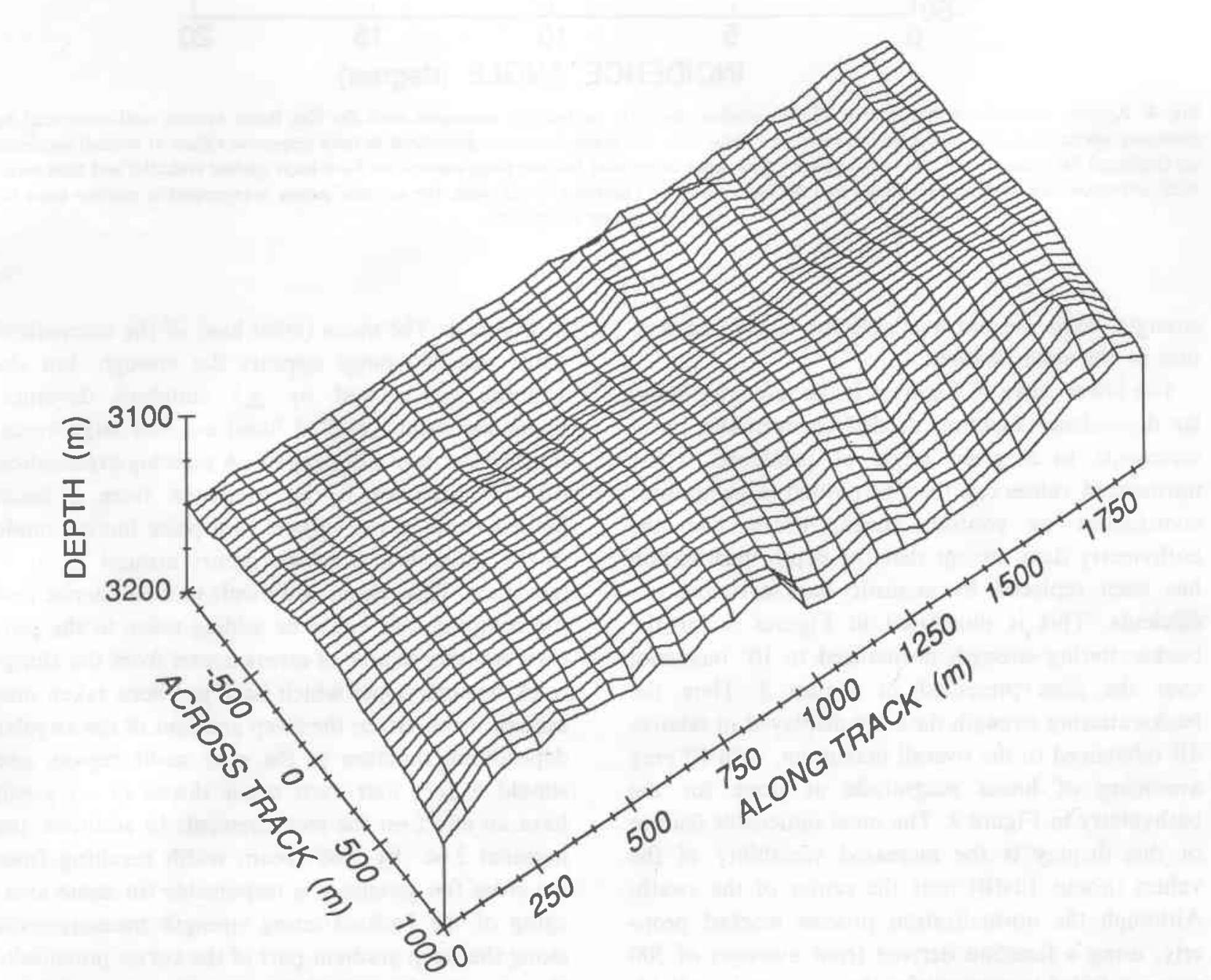

Fig 3. Segment of a bathymetric swath of Sea Beam data recorded over Magellan Rise. Data have been averaged over 10 pings for this display. The across-track scale is about half that of the along track, and a vertical exaggeration of 8.5 over the along-track scale has been used for depth. The center of the across-track axis corresponds to nadir. 


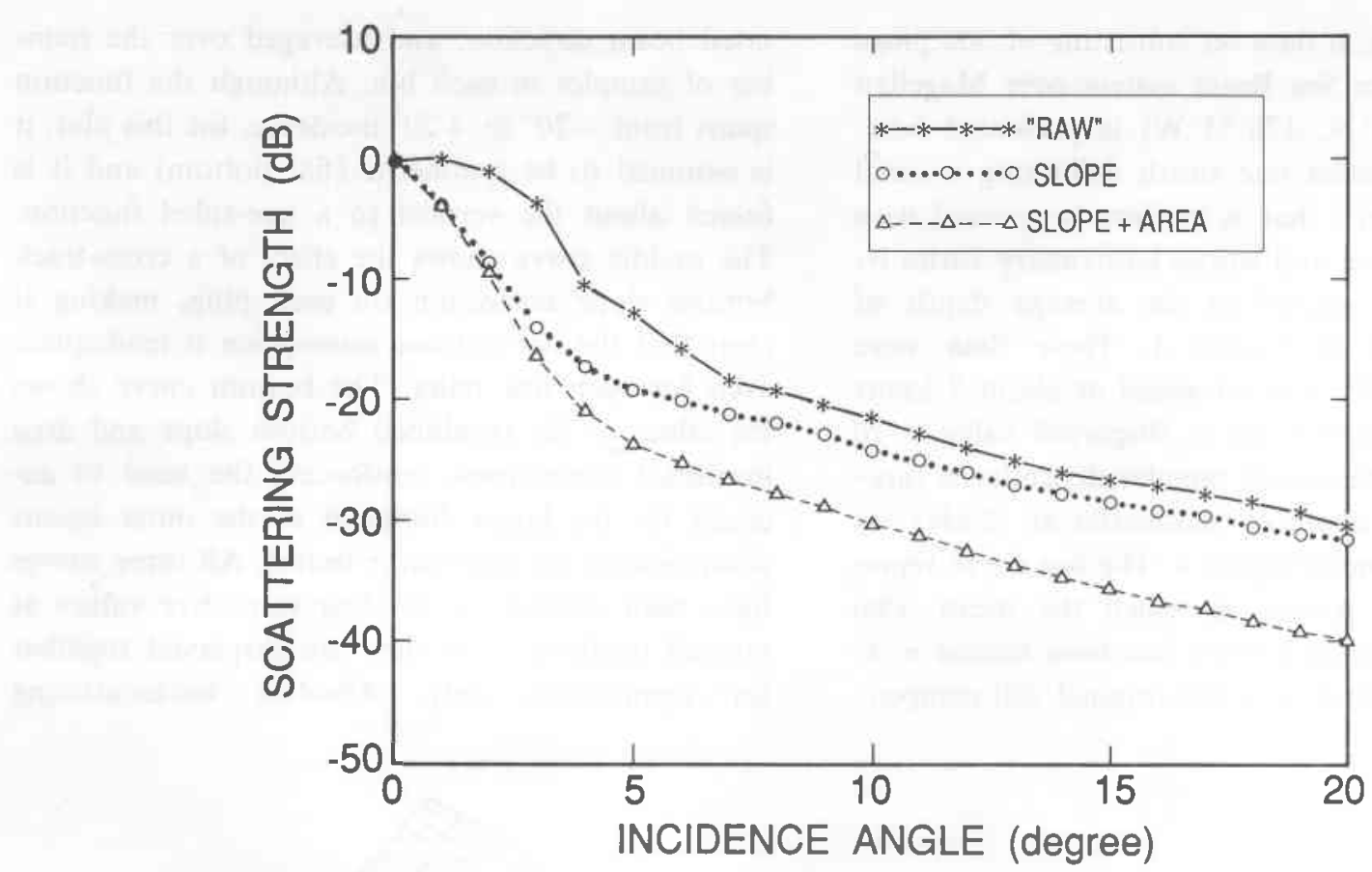

Fig. 4. Angular dependence function of $12 \mathrm{kHz}$ seafloor acoustic backscatter measured with the Sea Beam system, and computed by averaging normalized echo power accumulated in $1^{\circ}$ bins over 300 pings. 3 curves normalized to their respective values at normal incidence are displayed for comparison: 'raw' echo power (top), same curve after bottom slope corrections have been applied (middle) and final result with corrections for both bottom slopes and the area insonified (bottom). In all cases, the acoustic power is expressed in relative units for lack of system calibration.

strength levels are not available for lack of calibration of the sonar system.

The lower curve of Figure 4 is the reference angular dependence function needed to normalize measurements to a given angle of incidence. These normalized values can then be plotted in geographic coordinates as contour maps, much like the bathymetry data, except that the depth information has been replaced by acoustic backscattering coefficients. This is illustrated in Figures 5 for the backscattering strength normalized to $10^{\circ}$ incidence over the area presented in Figure 3. Here the backscattering strength data are displayed in relative $\mathrm{dB}$ referenced to the overall maximum, with 10 ping averaging of linear magnitude as done for the bathymetry in Figure 3. The most noticeable feature of this display is the increased variability of the values (about $10 \mathrm{~dB}$ ) near the center of the swath. Although the normalization process worked properly, using a function derived from averages of 300 pings, it failed to account for the variance in individual pings. This is best illustrated with a two-sided angular dependence of the normalized data as seen in Figure 6. The mean (solid line) of the normalized data over 300 pings appears flat enough, but the variance represented by \pm 1 standard deviation about the mean (dashed lines) exhibits large variations in the near nadir region. A possible explanation for this behavior is the transition from a beam limited mode in the vertical to a pulse limited mode in the outer beams which occurs around $3^{\circ}$ to $5^{\circ}$ incidence. This region is difficult to characterize and the computations could be adding noise to the process. Another source of errors comes from the alongtrack bottom slopes which have not been taken into account here. Given the steep gradient of the angular dependence function in the near nadir region, one should expect that even small slopes $\left(1-2^{\circ}\right)$ will have an effect on the measurement. In addition, the nominal $2.66^{\circ}$ by $2.66^{\circ}$ beam width resulting from the cross fan geometry is responsible for some averaging of the backscattering strength measurements along the steep gradient part of the curve, potentially changing its shape in the near nadir region (Ulaby, 1983). On the outer angles, beyond about $6^{\circ}$ incidence where measurements are pulse limited, small 


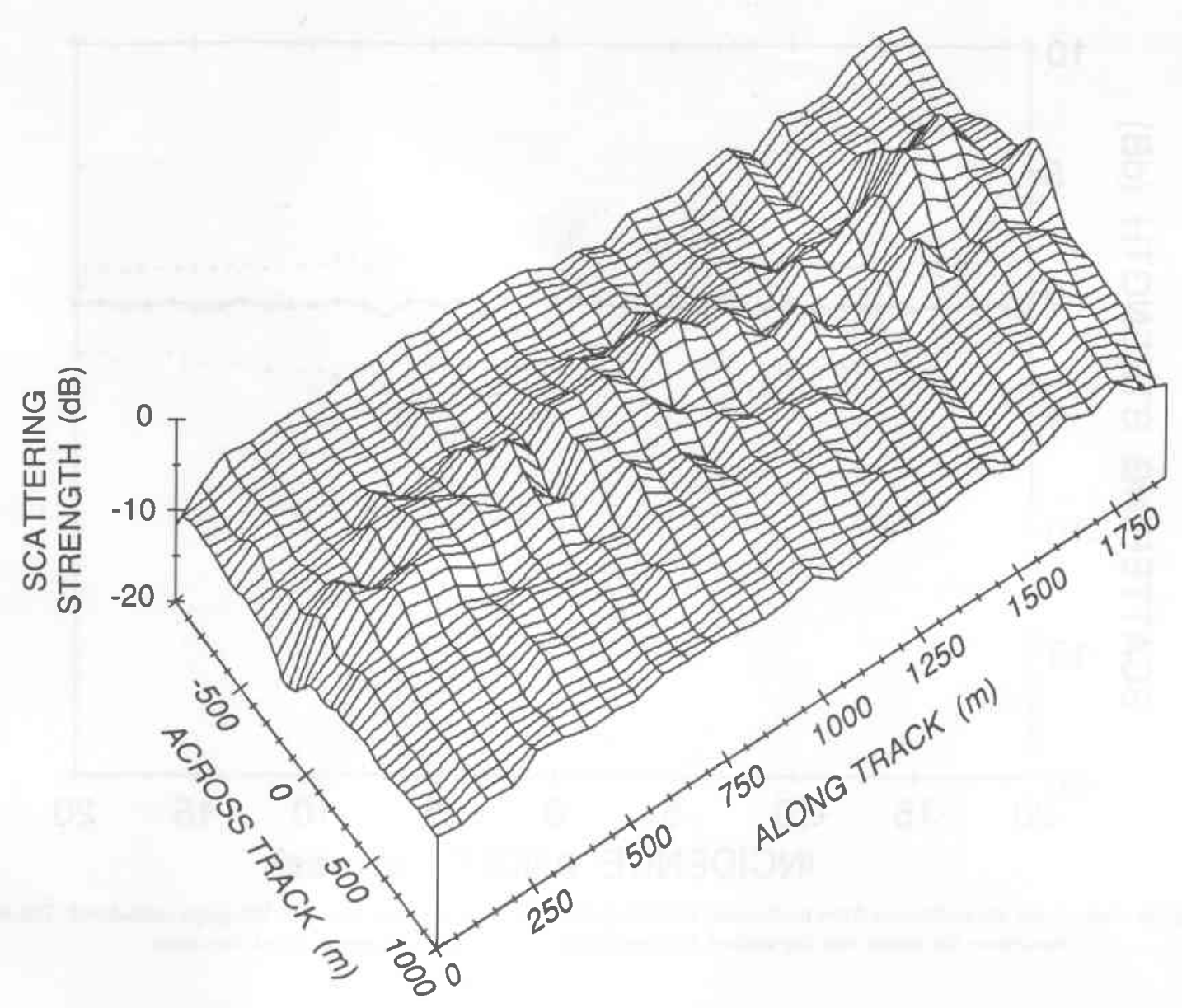

Fig. 5. Map of backscattering strength at $10^{\circ}$ incidence.

along-track slopes have less effect because the angular dependence curve is flatter.

\section{Acoustic Measurements with Bathymetric Sidescan Sonars}

Existing swath bathymetric sidescan sonars operate at acoustic frequencies ranging from 9 to $300 \mathrm{kHz}$, with transducers mounted in a vehicle towed behind a ship (Matsumoto, 1990; de Moustier, 1988). Unlike hull-mounted sonars, high frequency towed systems can be used at full ocean depth provided the tow cable is long enough to tow the sonar package a few tens to a few hundreds of meters above the bottom, and has enough bandwidth to accommodate the required data transmission rate. In the following, we concentrate on sonar systems towed about $100 \mathrm{~m}$ below the sea surface and capable of imaging to full ocean depth (e.g. SeaMarc II, Sys 09, Hawaii MR1, (Matsumoto, 1990; Shor, 1990)).

Depending on their acoustic power output, the systems operating at 9 to $12 \mathrm{kHz}$ achieve cross-track horizontal ranges of up to $15 \mathrm{~km}$ per side for sidescan data, and they obtain bathymetric coverage out to $60^{\circ}$ incidence or to the limit of the sidescan coverage, whichever is smallest. Simultaneous collection of sidescan data and bathymetry is a very important factor in the conversion of the typical qualitative acoustic image of the bottom into a quantitative acoustic backscatter map for seafloor classification applications. To this end, several methods have been developed to convert sidescan intensity data into meaningful quantitative values representative of particular bottom types. One approach is to use a statistical parameterization, by extracting the texture, the histogram of intensity or the 2-D spec- 


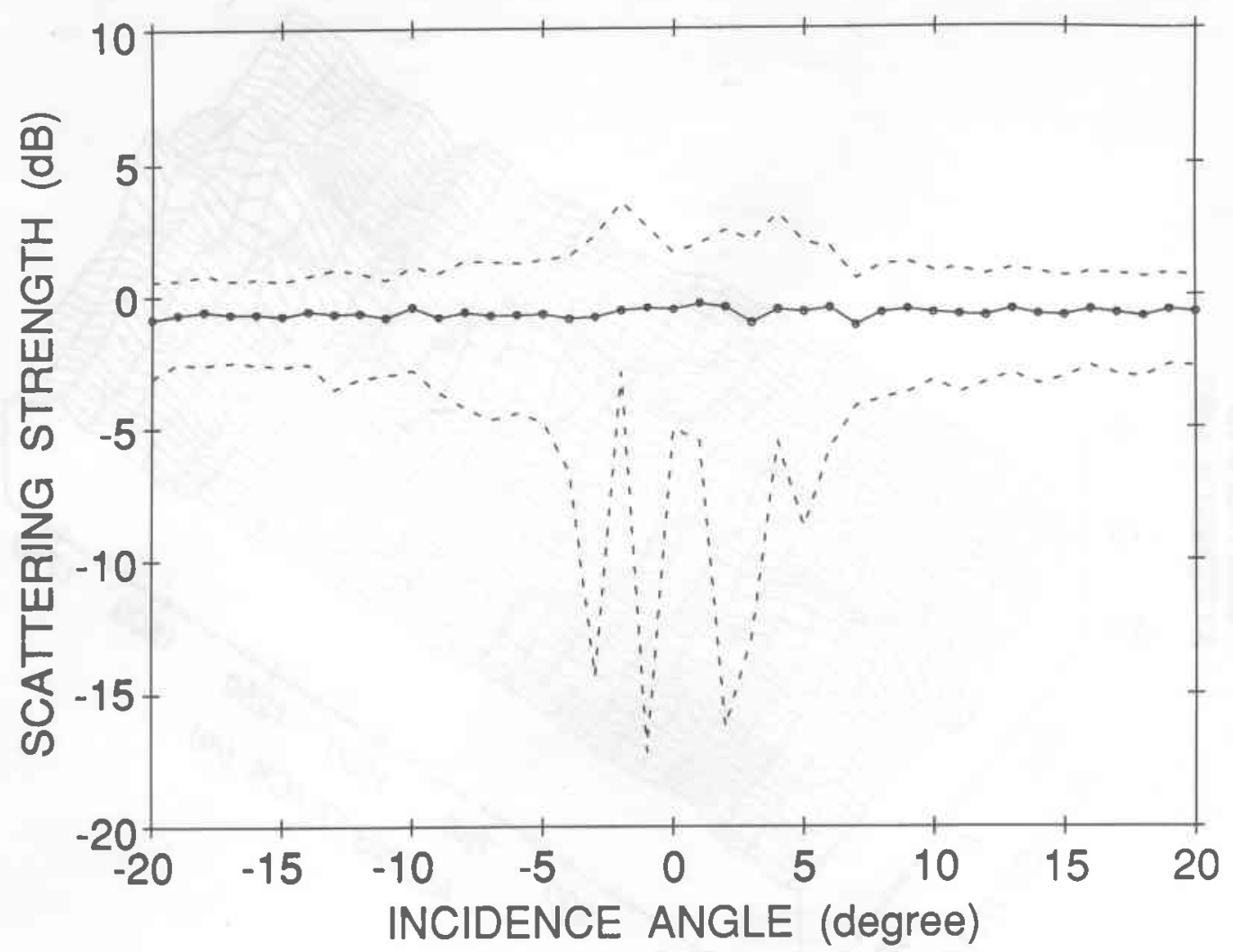

Fig. 6. Angular dependence curve derived from normalized scattering strength data averaged over the 300 pings considered. The solid line represents the mean and the dashed line represents \pm 1 standard deviation about the mean.

tral content from the acoustic image (e.g. Reut et al., 1985; Pace and Gao, 1988; Reed and Hussong, 1989). However, these statistical parameters are difficult to interpret and they must be validated by independent measurements such as bottom photographs or core samples before bottom classification can be considered.

Another approach is to convert the sidescan intensity data directly into physical acoustic parameters such as backscatter coefficients or acoustic impedance. As before, these maps may not necessarily give a unique answer for each bottom type because the backscattering process depends on a combination of surface roughness and material properties of the substrate that came into play as a sediment volume contribution or attenuation. However, backscattering strength maps are most useful when investigating the acoustical properties of the bottom and their effect on long range acoustic propagation. In the following, a technique to calculate backscatter coefficients from data measured with the
SeaMARC II system is presented. This technique is also applicable to data collected with other swath bathymetric sonar systems (e.g. System 09, System 120 , SeaMARC Izanagi, etc.).

For deep ocean survey work, the SeaMARC II system usually transmits a $1-2 \mathrm{~ms}$ pulse of $11 \mathrm{kHz}$ on port and $12 \mathrm{kHz}$ on starboard over a beam roughly $2^{\circ}$ wide 'along-track' and over $50^{\circ}$ wide 'across-track'. In that mode, the resulting sidescan image extends to a horizontal range of $5 \mathrm{~km}$ per side with a $5 \mathrm{~m}$ pixel side, and the bathymetry is limited to the lesser of $60^{\circ}$ incidence or $5 \mathrm{~km}$ horizontal range on each side. The $60^{\circ}$ limitation corresponds to the arrival of the first bottom multiple that most often destroys the differential phase measurements for echoes arriving from about $60^{\circ}$ from nadir. In SeaMARC II, the sidescan and bathymetric processing are handled by separate data acquisition systems aboard ship. Because of the separation of tasks, the sidescan data are displayed in real-time as intensity vs. horizontal distance perpendicular to the ship's 
track, where horizontal distances have been computed by assuming that the bottom is a ruled surface whose generating lines are horizontal and vary in elevation with the altitude of the vehicle. The resulting errors in pixel positions across the swath must be corrected during post-processing operations. A newer version of this system uses a single data acquisition system to record base-banded quadrature samples of the returns received at each transducer row, so that an integrated processing scheme can be implemented, where bathymetry and sonar imagery are always co-registered (de Moustier et al., 1991).

To illustrate the data processing steps needed to go from a real-time acoustic image to a backscatter map, we use data collected during a survey of the southern Endeavour Ridge segment within the Juan de Fuca Ridge system (Barone and Ryan, 1988). Figure 7 shows the acoustic image and the bathymetry of the area selected. The image covers about $120 \mathrm{~km}^{2}$ and the grey scale ranges from strong backscatter (black) to weak returns or acoustic shadows (white). The white stripes running $\mathrm{N}-\mathrm{S}$ are the result of navigation and attitude corrections applied to the data without filling in the gaps. The bathymetry has been hand-contoured at $100 \mathrm{~m}$ interval based on the raw values computed by the system. A small seamount cresting at $2600 \mathrm{~m}$ depth can be seen in the NE sector of the image and the ridge axis runs parallel to the NS direction. The 'concentric' contours in the NW corner represent the southern extent of the base of Endeavour Seamount.

This patch of seafloor was chosen because three parallel tracks intersect it (Figure 8), providing 3 different look angles on the terrain at both acoustic frequencies. The center image in Figure 8 corresponds to the unprocessed version of that displayed in Figure 7, and all three images have been cropped to retain only the overlapping region. The sequence of processing steps needed to convert these data to acoustic backscattering strength is briefly described below.

The first step consists in relocating pixels across the swath based on the bathymetry so as to un-do the flat bottom assumption that was used to display and record the image in real-time. This is a straightforward geometric correction wherever bottom echoes are non-ambiguous. In areas where echoes from different parts of the cross-track bathymetry profile arrive simultaneously at the vehicle, it is not possible to unravel the ambiguity and the corresponding pixels must be extrapolated to the nearest unambiguous samples. In areas of steep topography or large local relief, lateral errors in pixel location often exceed several hundred meters (Cervenka et al., 1990).

The next step involves the vehicle's attitude. Because the vehicle is towed approximately $400 \mathrm{~m}$ behind the ship and $100 \mathrm{~m}$ below the surface, its heading varies slightly from ping to ping and its track does not follow exactly the ship's heading. As a result, for any given ping, the cross-track sidescan line is not necessarily perpendicular to the ship's track, and there is a potential for pixel overlaps with previous pings. A computer mosaicing program uses the vehicle's attitude data (roll, yaw, pitch) and position, to correct such pixel location errors. Actually, yaw is usually less than $1^{\circ}$ on average and rarely requires corrections given that the horizontal beam width of the sonar is about $2^{\circ}$.

Once pixels have been assigned their best estimate of spatial coordinates, the intensity data need to be corrected for system related effects. During data acquisition, the system applies an angle variable gain to give a homogeneous appearance to the image across the swath. Therefore, the system's response (including beam pattern effects and variable gain functions, Figure 9) must be removed on a ping by ping basis from the intensity data, to obtain an angular dependence function of seafloor backscatter across the swath that is presumably dependent only on the scattering properties of the seafloor. Such angular dependence functions, for two distinct regions found in the data of Figure 7, are displayed in Figure 10. Each set of curves contains the $11 \mathrm{kHz}$ and the $12 \mathrm{kHz}$ components, and there are no significant differences between the results obtained at either frequency. However, these curves differ in their gradient from near nadir to $60^{\circ}$ incidence. The sharper gradient of Figure $7 \mathrm{~b}$ is indicative of an interface roughness dominated by small scale features comparable in size to a wavelength of the backscattered sound. In the $0-20^{\circ}$ incidence region, the shape of this curve is similar to that of the middle curve in Figure 4 which was clearly identified with a uniformly sedimented bottom. By contrast, the lower gradient of Figure $7 \mathrm{a}$ is representative of a surface with facets large compared to a wavelength, as would be found in a hummocky terrain. In both cases the general substrate descriptions of lava flow 

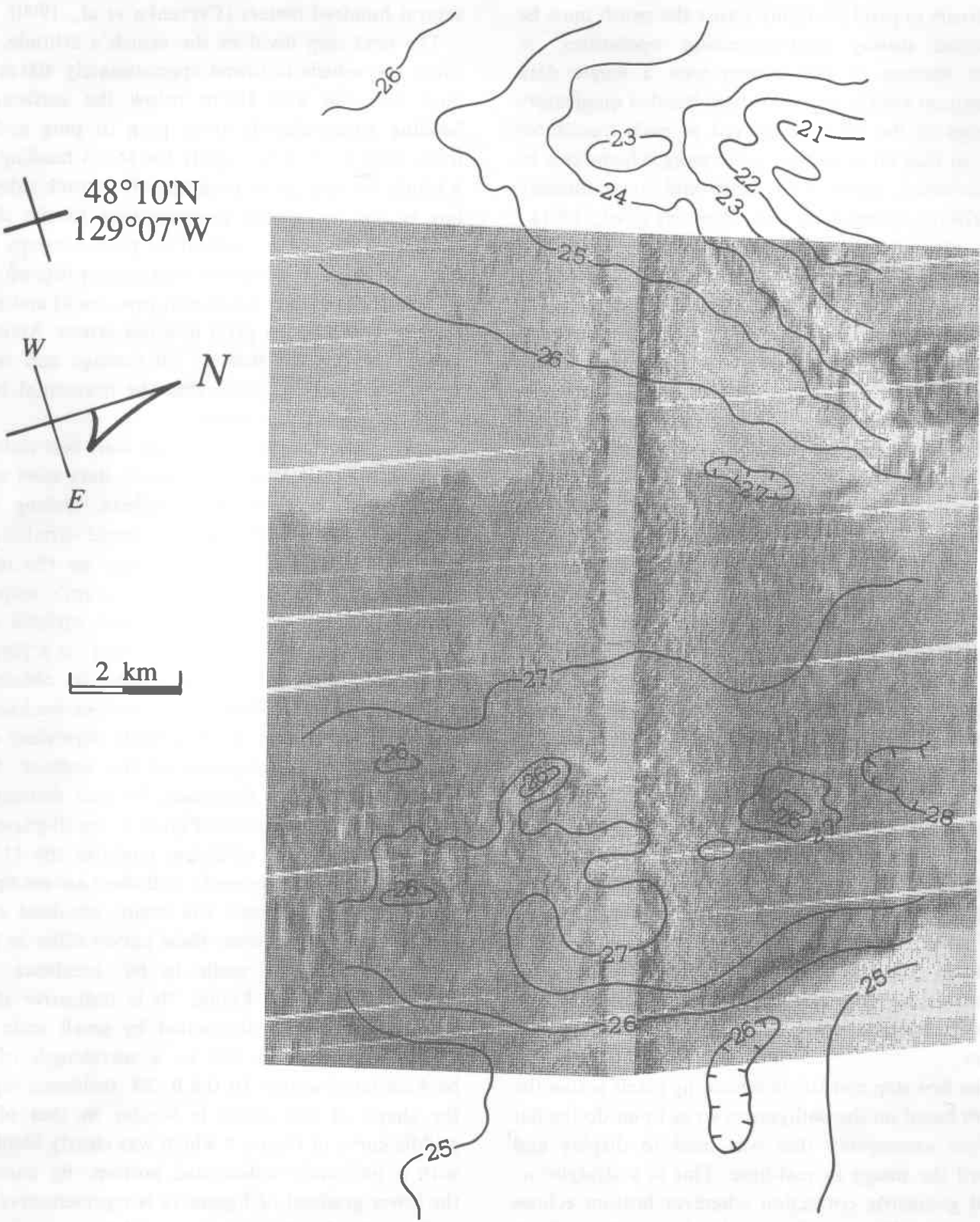

Fig. 7. Segment of a SeaMARC II swath recorded over the southern segment of the Endeavour ridge in the Juan de Fuca ridge system (near $48^{\circ} 12^{\prime} \mathrm{N}, 128^{\circ} 59^{\prime} \mathrm{W}$ ). The sidescan image is overlaid with hand-contoured bathymetry derived from the phase data and plotted at $100 \mathrm{~m}$ intervals. 


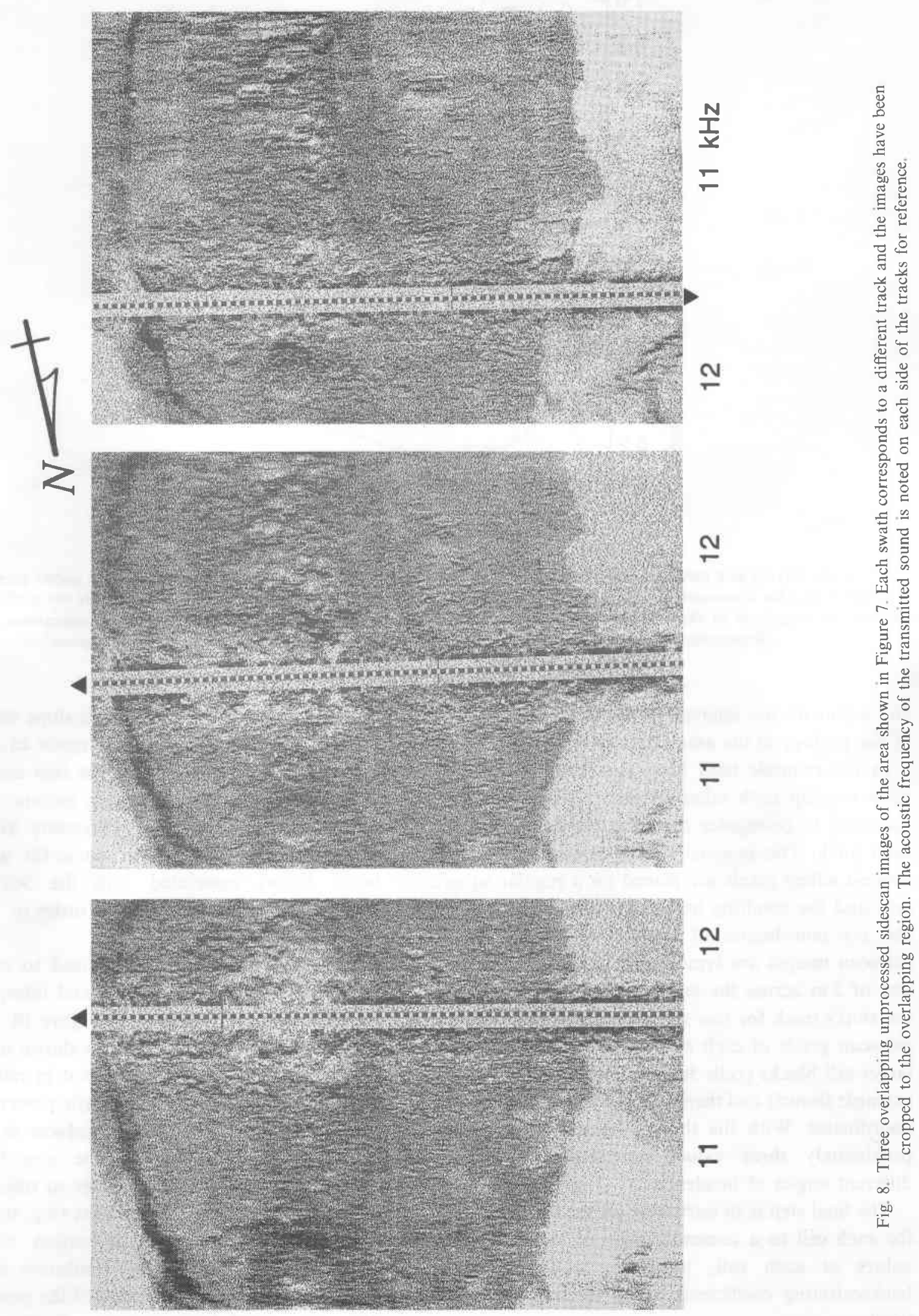



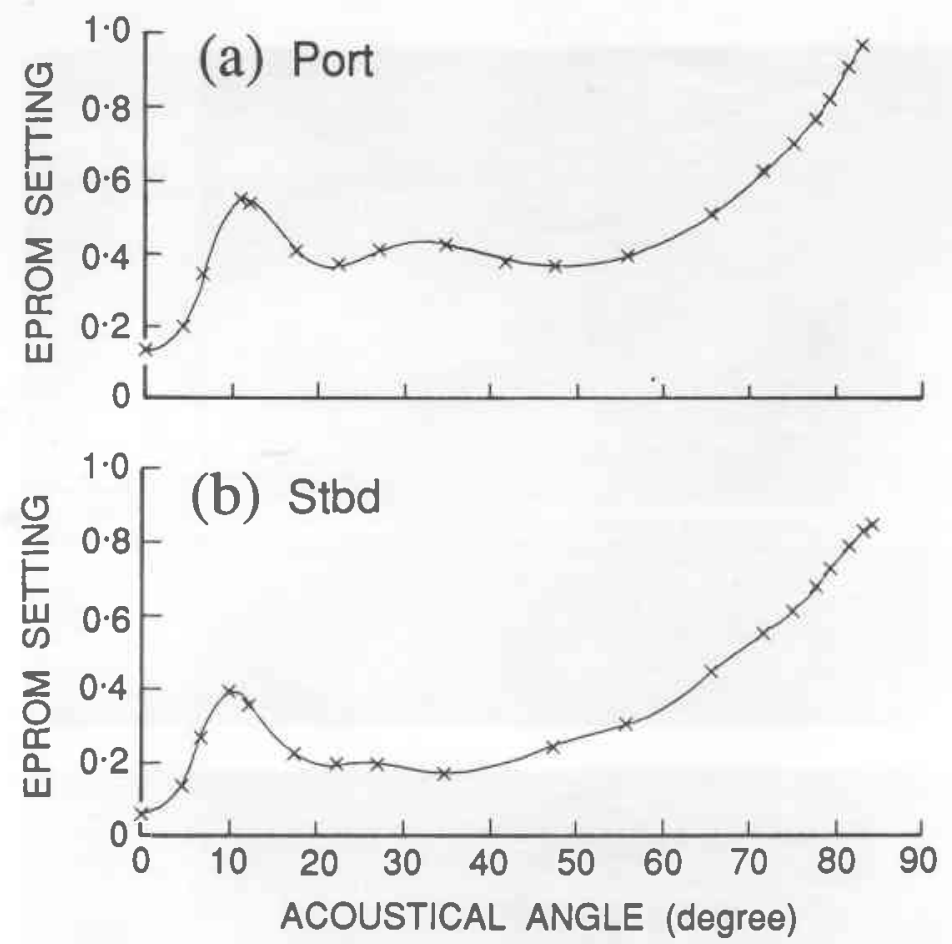

Fig. 9. Angle varying gain used by SeaMARC II to correct for system related effects (array beam patterns and system gains). Under the assumption of a flat horizontal bottom, acoustic angle refers to the angle of incidence. The EPROM setting are the coefficients used to multiply the magnitude of the echo received at a given angle in order to give the image a more uniform appearance across track. Representative samples $(x)$ of the gain tables have been fitted with a spline for display purposes.

and sediments are inferred from reported knowledge of the geology in the area (Barone and Ryan, 1988).

In the example used here, the three swaths partially overlap each other (Figure 8), it is therefore necessary to co-register the corresponding pixels for each track. This is usually done through a gridding process where pixels are placed on a regular square grid and the resulting images are overlaid based on the $x-y$ coordinates of each pixel. SeaMARC II sidescan images are typically displayed with a pixel size of $5 \mathrm{~m}$ across the swath and about $30 \mathrm{~m}$ along the ship's track for tow speeds of 8 knots. Here, the sidescan pixels of each swath are first grouped into larger cell blocks (cells $50 \mathrm{~m} \times 50 \mathrm{~m}$ were used in the example shown) and then overlaid based on their $x-y$ coordinates. With the three swaths, this yields approximately three values representing the three different angles of incidence.

The final step is to normalize all the measurements for each cell to a common angle of incidence. The values at each cell, therefore, would represent backscattering coefficients at up to three angles of incidence.
Here, the athwartships bottom slope was assumed to be $0^{\circ}$, thus no attempt was made to correct the angle of arrival to determine the true angle of incidence based on the bathymetry, because the resolution of the SeaMARC II bathymetry available for this survey was deemed too coarse for such corrections. Errors associated with the SeaMARC II bathymetry are typically on the order of 2 to $3 \%$ of the water depth.

All values have been normalized to an angle of incidence of $45^{\circ}$, using polynomial interpolation or extrapolation on the curves of Figure 10. The resulting backscatter coefficient map is shown in Figure 11 where measurements are presented in relative units, for lack of information on acoustic power and sensitivity of the SeaMARC II transducer arrays. Four classes are distinguishable in the map, however to unequivocally assign these classes to substrate types would require visual observations (e.g. bottom photographs or video) and sub-bottom sampling or imaging (e.g. core, or high-resolution sub-bottom profiling). Based on knowledge of the geology in the survey area and the proximity of the ridge, these 


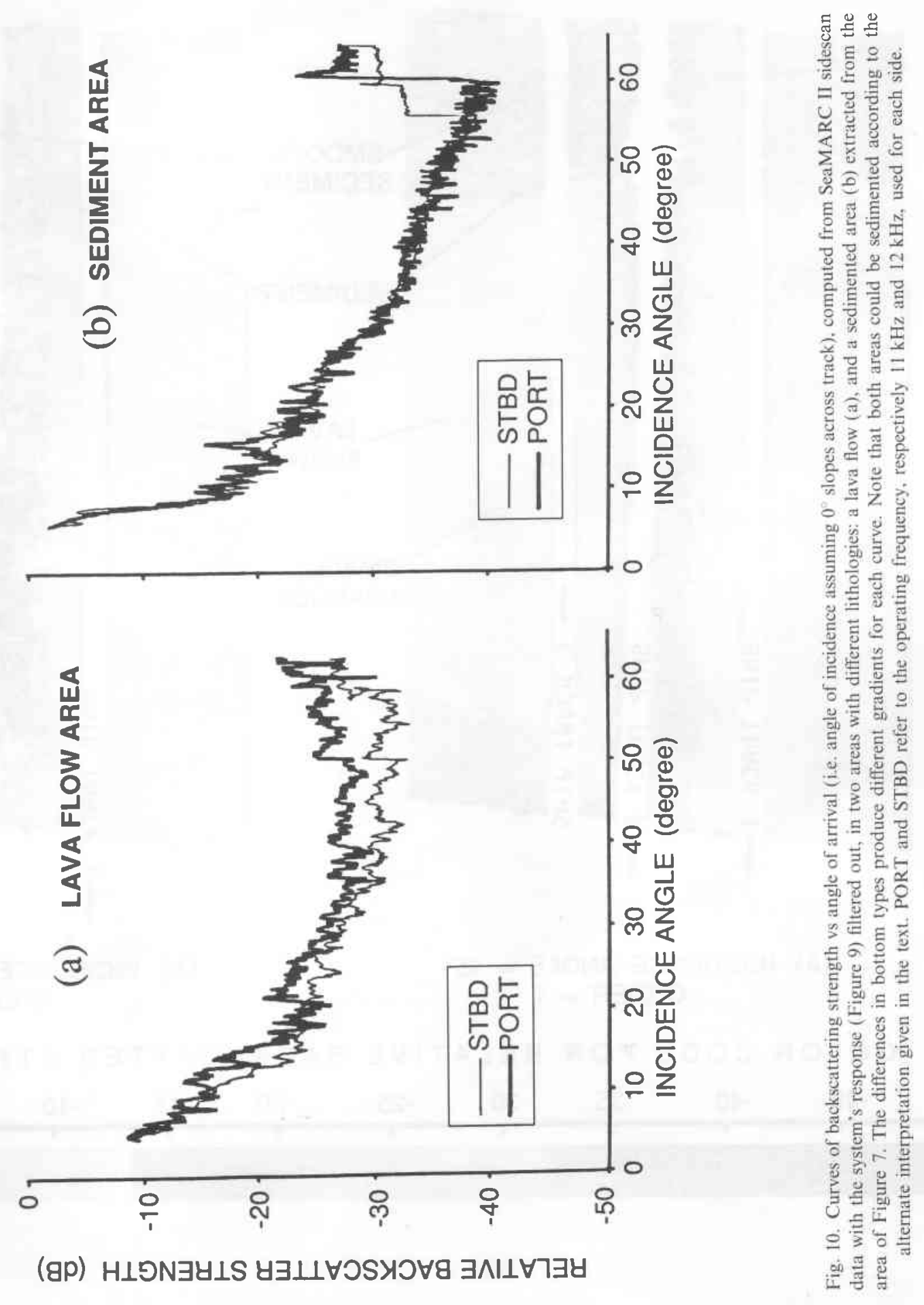




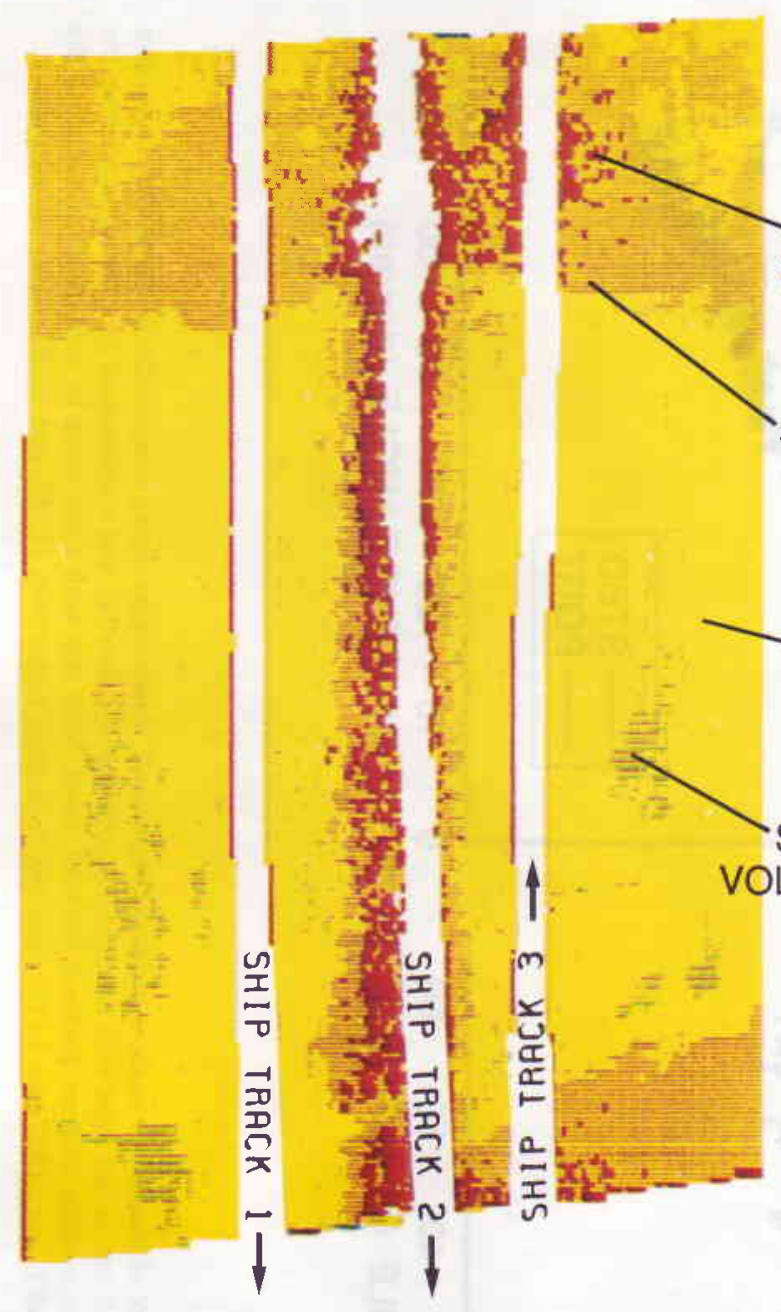

(a) INCIDENCE ANGLE $=45^{\circ}$ ORDER $=1$

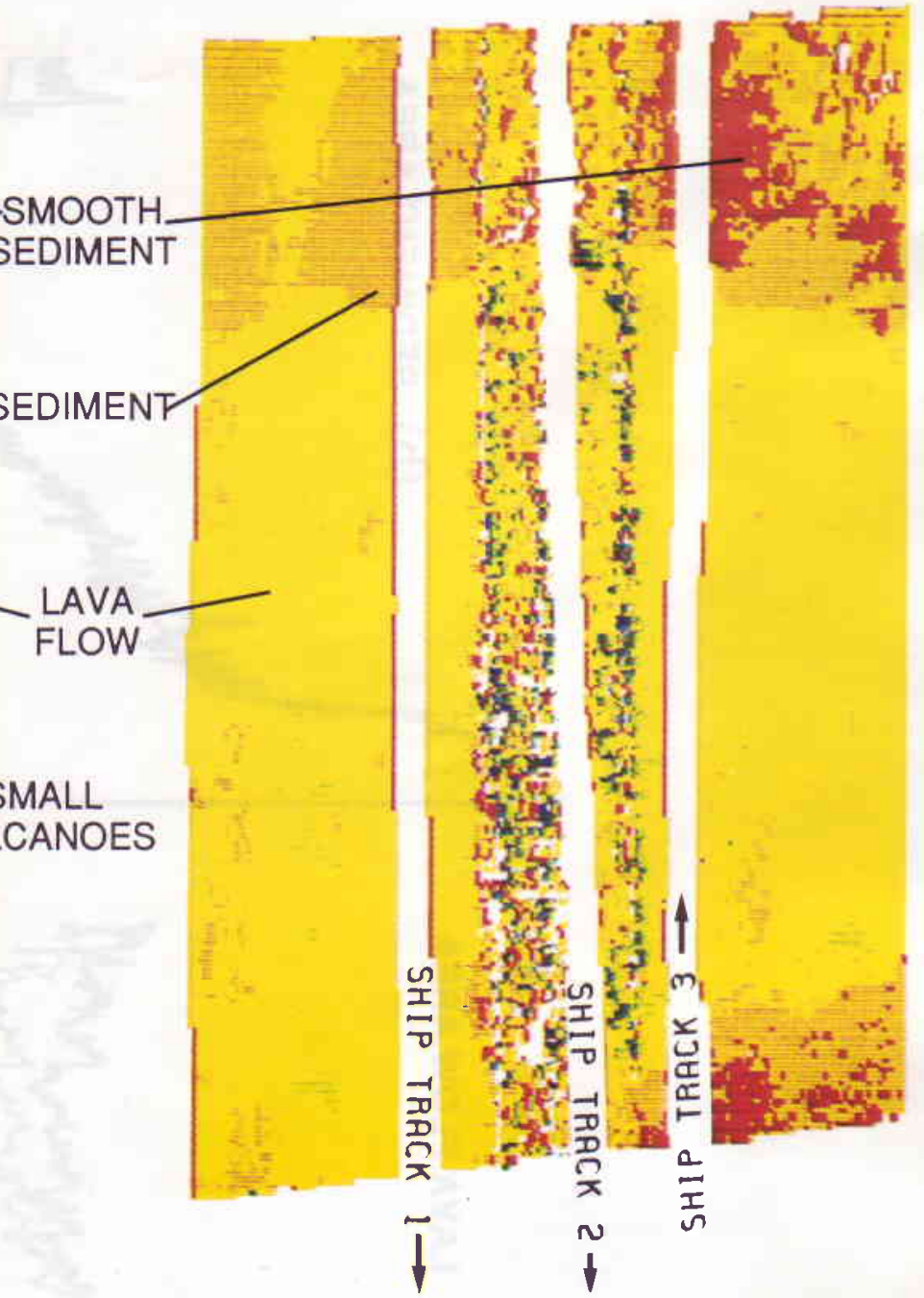

(b) INCIDENCE ANGLE $=45^{\circ}$ ORDER $=2$

COLOR CODE FOR RELATIVE BACKSCATTER STRENGTH (dB)

\begin{tabular}{llllllllllll}
-50 & -45 & -40 & -35 & -30 & -25 & -20 & -15 & -10 & -5 & 0 & +5 \\
\hline
\end{tabular}

Fig. 11. Maps of $11-12 \mathrm{kHz}$ seafloor backscatter as would be measured at 45 incidence over the area covered by the three tracks of Figure 8. Backscattering strength is expressed in relative units for lack of system calibration. The curves of Figure 10 are used to interpolate/extrapolate backscattering strengths measured at various angles to a nominal $45^{\circ}$ incidence with a 1 st order polynomial (a), or a 2 nd order polynomial (b). Pixel size is $50 \mathrm{~m}$. 
classes could possibly represent lava with two types of surface roughness: e.g. hummocky volcanic substrate with new flows partially covering it and yielding a smoother relief as viewed at $11-12 \mathrm{kHz}$; and two types of surface roughness in sedimented areas: e.g. at the foot of Endeavour Seamount where complete sediment coverage is assumed. Alternatively, the apparent differences in surface roughness could be simply due to variations in the thickness of sediments overlying lava flows. A thin sediment drape (1-2 $\mathrm{m}$ thickness) could be essentially transparent to the impinging $11-12 \mathrm{kHz}$ sound waves, and strong backscatter would then be due to the underlying basalt. Although we have no photographic evidence to substantiate any of these hypotheses, the different lava roughnesses are partly corroborated by results from a $30 \mathrm{kHz}$ sidescan survey of this area with the SeaMARC I sonar system, which indicate that the small volcano in the image is a 'young' structure with recent lava flows around it covering older rougher terrain (Barone, pers. commun.).

The $0^{\circ}$ bottom slope assumption made in the previous step is usually not valid and, as for the multibeam acoustic data presented above, angles of arrival must be converted to angles of incidence by taking the cross-track bottom slopes into account. Here again, high-resolution bathymetry is required for this correction. In addition, navigation errors will cause misregistration of pixels between images. A third type of error comes from insufficient knowledge of the system's response, particularly its beam patterns, and from only approximate corrections for raybending effects through the water column. Such corrections are implicitly taken into account in the table used to convert differential phase to acoustic angle of arrival. We believe that a combination of these factors is responsible for the speckle-like errors seen in Figure 11.

With better navigation (GPS) and higher resolution bathymetry, the accuracy of the co-registration will certainly improve. From recent survey results with the newly developed System 09, depth accuracy of about $1 \%$ of water depth seems attainable provided raybending corrections are applied. Likewise, calibration of the acoustic response of these systems will permit better normalization of the measurements and derivation of absolute values.

\section{Conclusions}

Over the past decade, advances in sonar systems designed for swath bathymetry surveys have made it possible to map several thousand square kilometers of ocean floor per day with a depth resolution usually better than $1 \%$ of the water depth and samples co-registered and spaced at intervals of 3 to $5 \%$ of the water depth within each swath. The foregoing discussion indicates that these same systems can be used for remote acoustic classification of the seafloor if the echoes received are corrected to absolute acoustic backscattering coefficients. The key parameters for these corrections are: (1) bathymetric slopes to take the angular dependence of seaffoor acoustic backscatter into account, (2) the system's transfer function including transmit/receive patterns, source level and sensitivity that are needed to obtain absolute values, and (3) the positional accuracy of the samples as it influences along-track slope calculations as well as swath to swath data registration. As newer systems are introduced, and with the advent of 24 hours/day GPS navigation, items 1 and 3 will continue to improve. Item 2 remains a difficult problem as the acoustic transducer arrays used for deep ocean work, whether hull-mounted or installed in a tow-vehicle cannot be calibrated in a tank facility, and are difficult to calibrate in the field (de Moustier et al., 1990).

\section{Acknowledgements}

This work was funded by the Office of Naval Research whose support is graciously acknowledged. ONR contract \# N00014-90-J-1062. In addition, we wish to thank Dale Chayes for helpful review comments and J. Griffith for the art work.

\section{References}

Alexandrou, D., and de Moustier, C., 1988, Adaptive noise cancelling applied to Sea Beam sidelobe interference rejection, IEEE J. Oceanic Eng., 13 (2), 70-76.

Alexandrou, D., de Moustier, C., and Haralabus, G., 1992, Evaluation and verification of bottom acoustic reverberation statistics predicted by the point scattering model, J. Acoust. Soc. Am., 91 (3), 1403-1413.

Barone, A. M., and Ryan, W. B. F., 1988, Along-Axis Variations within the Plate Boundary Zone of the Southern Segment of the Endeavour Ridge, J. Geophys. Res., 93 (B7), 7856-7868.

Blackinton, J. G., Hussong, D. M., and Steenstrup, J., 1991, Seafloor Cable Surveys, Sea Technology, 32 (7), 33-39. 
Cervenka, P., de Moustier, C., and Lonsdale, P. F., 1990, Pixel relocation in SeaMARC II sidescan sonar images based on gridded Sea Beam bathymetry, EOS, Trans. Amer. Geophys. Union, 71 (43), 1407-1408. 1990.

Davis, E. E., Currie, R. G., Sawyer, B. S., and Kosalos, J. G., 1986, The use of swath bathymetric and acoustic image mapping tools in Marine Geoscience, Mar. Tech. Soc. J. 20 (4), 17-27.

Hammerstad, E., Pohner, F., Parthiot, F., and Bennett, J., 1991, Field testing of a new deep water multibeam echo-sounder, Proc. IEEE Oceans' '91, 2, 743-749.

Jackson, D. R., Winebrenner, D. P., and Ishimaru, A., 1986, Application of the composite roughness model to high-frequency bottom backscattering, J. Acoust. Soc. Am., 79 (5), 1410-1422.

Ulaby, F. T., Allen, C. T., and Fung, A. K., 1983, Method for retrieving the true backscattering coefficient from measurements with a real antenna, IEEE Trans. on Geoscience and Remote Sensing, GE-21 (3), 308-313.

Matsumoto, H., 1990, Characteristics of the SeaMARC II phase data, IEEE J. Oceanic Eng., 15 (4), 350-360.

Mitchell, N. C., and Somers, M. L., 1989, Quantitative Backscatter Measurements with a Long-Range Side-Scan Sonar, IEEE J. Oceanic Eng., 14 (4), 368-374.

de Moustier, C., 1986-a, Approaches to acoustic backscattering measurements from the deep seafloor, Symposium on Current Practices and New Technology in Ocean Engineering, Am. Soc. Mech. Eng., OED 11, 137-143. (Reprinted in Trans. of the ASME, $J$. Energy Resources Tech., 110, 77-84, 1988).

de Moustier, C., 1986-b, Beyong bathymetry: mapping acoustic backscattering from the deep seafloor with Sea Beam, $J$. Acoust. Soc. Am., 79 (2), 316-331. de Moustier, C., 1988, State of the art in swath bathymetry survey systems, International Hydrographic Review, Monaco, LXV (2), 25-54.

de Moustier, C., Matsumoto, H., and Shor, A. N., 1990, Open ocean acoustic calibration of the SeaMARC II bathymetry sidescan sonar system, Proc. IEEE Oceans '90, Washington D.C., 523-527.

de Moustier, C., and Alexandrou, D., 1991, Angular dependence of $12 \mathrm{kHz}$ seafloor acoustic backscatter, J. Acoust. Soc. Am., 90 (1), 522-531.

de Moustier, C., Masnadi-Shirazi, M. A., and Cervenka, P., 1991, Integrated processing for bathymetry and sidescan data in swath bathymetry side-looking sonars, EOS, Trans. Am. Geophys. U., 72 (44), 249-250.

National Research Council, Marine Board, 1989, Our seabed frontier, challenges and choices, National Academy Press.

Ol'shevskii, V. V., 1967, Characteristics of sea reverberation, Consultant Bureau, Plenum Press, N.Y.

Pace N. G., and Gao, H., 1988, Swathe seabed classification, IEEE J. Oceanic Eng., 13 (2), 83-89.

Reed, T. B. IV, and Hussong, D. M., 1989, Quantitative Analysis of SeaMARC II Side-Scan Sonar Imagery, J. Geophys. Res., 94 (B6), 7469-7490.

Reut, Z., Pace, N. G., and Heaton, M. J. P., 1985, Computer Classification of Sea Beds by Sonar, Nature 314, 426-428.

Shor, A. N., 1990, SeaMARC II seafloor mapping system: Seven years of Pacific research, Proc. Pacific Rim Congress 90, 3, $49-59$.

Stanton, T. K., 1984, Sonar Estimates of Seafloor Microroughness, J. Acoust. Soc. of Am., 75 (3), 809-818. 